\title{
Method for vibro-acoustic signal processing for detection of nascent defect of mechanical assemblies
}

\author{
Lange $\mathrm{P}$. \\ Department of measuring technology \\ Samara State Technical University \\ Samara, Russia \\ lange_distance@mail.ru
}

\author{
Yaroslavkina E. \\ Department of measuring technology \\ Samara State Technical University \\ Samara, Russia \\ makarovak@inbox.ru
}

\begin{abstract}
In the article, the method of early diagnostics of nascent defects of mechanical assemblies (friction nodes, bearing pairs, gears) is considered by on-line determination of high-order statistical moments of the vibro-acoustic signal. To determine such statistical moments, a parabolic spline approximation of the discrete signal values is used. The spline approximation coefficients are determined at each discrete sampling interval by digital filter expressions. Expressions for the second, third and fourth statistical moments of the vibroacoustic signal are determined using its parabolic and cubic spline approximation. The signal processing is simple enough and can be implemented in a microprocessor controller. The characteristics of the proposed method are determined by an example of processing a vibro-acoustic signal of a harmonic shape. It is shown that with a relatively rare signal sampling, the error in determining the dispersion and the fourth statistical moment (Excess) of the signal is rather small. The use of a parabolic spline approximation of a vibro-acoustic signal makes it possible to significantly reduce the frequency of the signal sampling and to reduce the amount of data needed to determine the statistical characteristics of the signal.
\end{abstract}

Keywords - nascent defect, vibro-acoustic signal, signal processing, excess of signal distribution, method of excess signal determination

\section{INTRODUCTION}

Today problems of early diagnostics of nascent defects (scuffing, seizing, etc.) of mechanical assemblies and units working in a stressed mode are coming to the fore $[1,2]$. The methods of detecting such defects were classified in [2, $3,12,30]$. A number of researchers have investigated the effect of acoustic emission in the detection of bearing defects [13-16, 18]. In particular, it was noted that the seizing of gear and bearing pairs is one of the most common types of failure at high temperatures with a lack of lubrication. The vibration technique has been investigated and is established as a diagnostic technique for rotating tools in machinery in $[5-7,12,17,19]$. The use of acoustic emission in the rail track fault detection was investigated in [23], in powerful mechanical units - in [21, 24], in gearboxes - in $[20,22,25]$. A number of researches present vibration analysis for early fault detection [8, 9]. The use of multivariate statistical analysis of vibration signals for the detection of localized faults in two-stage helical gears was investigated and explored in [10, 26].

With use of vibro-acoustic diagnostics methods, the initial phase of a defect is difficult to diagnose, since the spectral pattern of the vibro-acoustic signal in this phase practically does not change. In this case, for the early diagnoses, methods for determining the excess, associated with the fourth-order statistical moment of vibro-acoustic signal, can be applied [4], which makes it possible to diagnose the wear of a gear or bearing pair at an early stage of its occurrence fairly well.

For the diagnostics purposes, the distribution curve $p(x)$ of the acoustic signal $x(t)$ can be used. At a normal state, this curve is two-mode. When defect occurs, the curve $p(x)$ changes significantly and becomes unimodal, which indicates the growth of noise components. The degree of "fineness" or "flatness" of the distribution law is customarily characterized by the magnitude of the Excess (Ex).

Figure 1 shows the dependence of the excess magnitude on time of operation of the car rear axle in view of lubricant deficit.

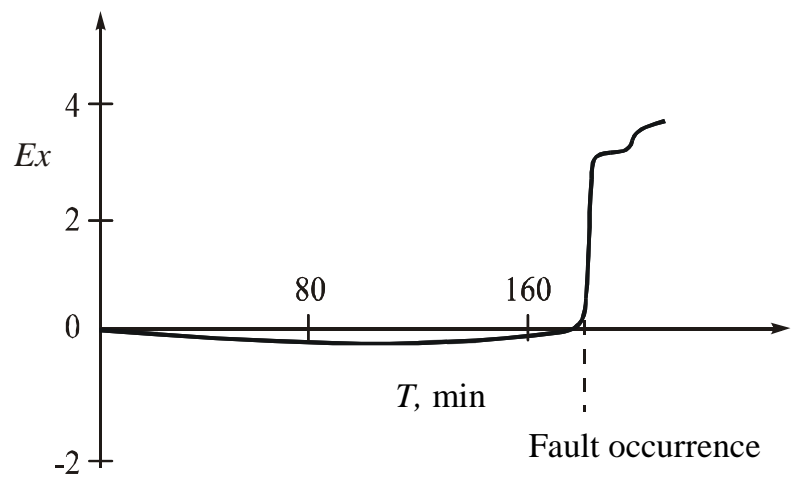

Fig.1. Dependence of the $E x$ of the acoustic signal on the time of operation of a car rear axle.

Regardless of the load degree, the Ex is practically equal to zero, and increases only when a defect magnitude increases dramatically, which allows one to carry out a reliable early diagnosis of the rear axle. 
Approximately the same way is used to diagnose the scuffing of the crankshaft bearings of a power unit by determining the excess.

Statistical methods of other orders are also used to diagnose defects in mechanical assemblies. For example, such defect as a lateral gap in the gearing of the reducer can be diagnosed by the dispersion of a vibro-acoustic signal. The larger the gap in meshing is, the greater the dispersion in both the wide frequency band and the octave bands of vibro-acoustic signal is.

\section{DESCRIPTION OF MATHEMATICAL METHOD FOR THE ON- LINE EXCESS DETERMINATION OF THE VIBRO-ACOUSTIC SIGNAL}

Let us consider the algorithm for the moment analysis of vibro-acoustic signals.

In practice, the central moment of the $k$-th order for a stationary ergodic random signal is usually determined by its temporal realization:

$$
\mu_{x k}=\frac{1}{T} \int_{0}^{T} x_{0}^{k}(t) d t
$$

where $x_{0}(t)$ is a centered signal,

$T$ is the period of its processing.

As is well known, the centered signal is determined by subtracting the expected value from the signal.

Vibro-acoustic signals have a limited frequency spectrum, so the algorithms for determining the highest moments can be estimated using the example of a harmonic signal:

$$
x=\cos \omega t .
$$

Taking into account that the measurement of the signal with equal probability can be carried out at any time, which may be not synchronized with the signal frequency, the law of distribution of the signal value is determined by the expression [11]:

$$
p(x)=\frac{1}{\pi \sqrt{1-x^{2}}} .
$$

Taking into account that the normalized signal $x$ varies in the range of $(-1,+1)$, the dispersion of the signal is:

$$
D_{x}=\int_{-1}^{1} x^{2} p(x) d x=\frac{1}{\pi} \int_{-1}^{1} \frac{x^{2} d x}{\sqrt{1-x^{2}}}=1 / 2 .
$$

Accordingly, the third central moment is given by:

$$
M_{3}=\frac{1}{\pi} \int_{-1}^{1} \frac{x^{3} d x}{\sqrt{1-x^{2}}}=0
$$

and the fourth central moment is given by expression:

$$
M_{4}=\frac{1}{\pi} \int_{-1}^{1} \frac{x^{4} d x}{\sqrt{1-x^{2}}}=3 / 8=0.375 .
$$

These moments, in view of the stationarity and ergodicity of the signal, can be determined with the help of (1), and their numerical values in this case coincide with the values (3) - (5).
Let us consider the problem of determining these moments proceeding from discrete values of the signal.

Within the sampling intervals, the signal can be reconstructed with a certain error by an approximating function. In this case, the coefficients of the approximating function can be used to determine the moments (3)-(5).

Let us consider the possibility of using an approximating parabolic spline function for this purpose, which is described on the $n$th sampling interval by an expression

$$
x(t)=a_{2}[n] t^{2}+a_{1}[n] t+a_{0}[n] .
$$

Coefficients $a_{2}[n], a_{1}[n], a_{0}[n]$ are determined by the corresponding expressions of digital spline filters.

For example, for a five-point parabolic spline filter these expressions are determined by the relations [27]

$$
\begin{aligned}
& a_{0}[n]=1 / 16(-x[n-2]+4 x[n-1]+10 x[n]+4 x[n+1]-x[n+2]), \\
& a_{1}[n]=1 / 8 t_{d}(x[n-2]-6 x[n-1]+6 x[n+1]-x[n+2]), \\
& a_{2}[n]=1 / 16 t_{d}^{2}(-x[n-2]+7 x[n-1]-6 x[n]-6 x[n+1]+ \\
& +7 x[n+2]-x[n+3]),
\end{aligned}
$$

where $t_{d}$ is the signal sampling interval,

$x[n]$ is the discrete value of the signal at the $n$-th point in time.

Accordingly, for a cubic spline filter, analogous expressions for the spline-function coefficients are determined by the relations [28]

$$
\begin{aligned}
& a_{0}[n]=\frac{1}{36}(-x[n-2]+4 x[n-1]+30 x[n]+4 x[n+1]-x[n+2]), \\
& a_{1}[n]=\frac{1}{12 t_{d}}(x[n-2]-8 x[n-1]+8 x[n+1]-x[n+2]), \\
& a_{2}[n]=\frac{1}{12 t_{d}^{2}}(-x[n-2]+10 x[n-1]-18 x[n]+10 x[n+1]- \\
& -x[n+2]), \\
& a_{3}[n]=\frac{1}{36 t_{d}^{3}}(x[n-2]-11 x[n-1]+28 x[n]-28 x[n+1]+ \\
& \quad+11 x[n+2]-x[n+3]) .
\end{aligned}
$$

The parabolic and cubic spline functions, as is well known, have no discontinuities at the boundaries of the sampling intervals with respect to the 0th and 1st derivatives. So the use of spline- function approximating practically does not cause the appearance of higher harmonics in the signal spectrum reconstructed by such approximations.

It should be noted that the digital filters used to determine the spline-approximation coefficients have certain smoothing properties. For example, the frequency response of a digital filter that determines coefficient $a_{0}[n]$ of a parabolic spline approximation in (6) is given by:

$$
A_{p}\left(f_{1}\right)=\frac{5+4 \cos \left(2 \pi f_{1}\right)-\cos \left(4 \pi f_{1}\right)}{8},
$$

where $f_{1}$ is the relative frequency of the signal, 
$f_{1}=1 / N, \quad N$ - number of samples on the period of the signal [29].

A similar expression for the coefficient $a_{0}[n]$ of the cubic spline approximation in (7) has the form:

$$
A_{c}\left(f_{1}\right)=\frac{15+4 \cos \left(2 \pi f_{1}\right)-\cos \left(4 \pi f_{1}\right)}{18} .
$$

The graphs of the frequency responses (8) and (9) are shown in Fig. 2.

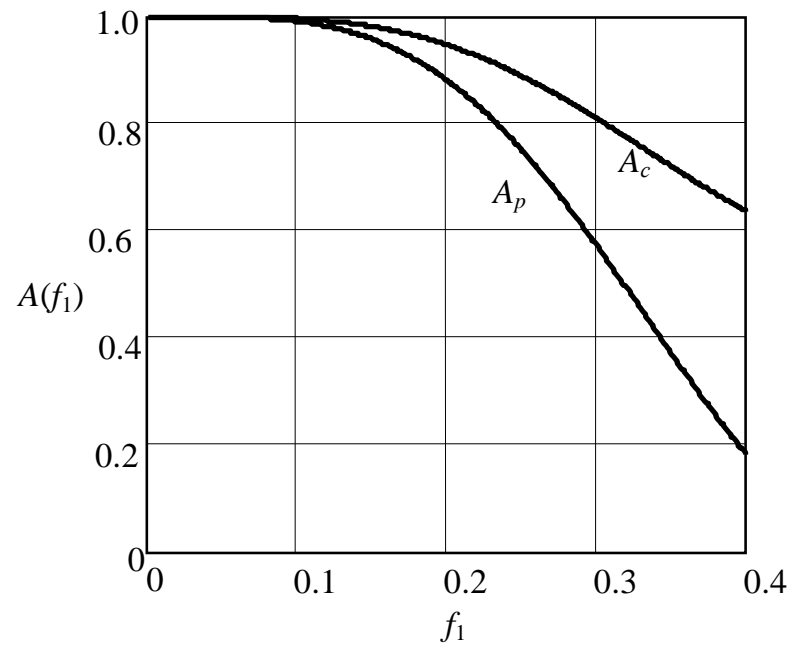

Fig. 2. Graphs of frequency responses $A_{p}\left(f_{1}\right) A_{c}\left(f_{1}\right)$ of parabolic and cubic spline filters, respectively.

It can be seen from Fig. 2 that digital spline filters suppress high-frequency interference, which is a valuable property of theirs.

The expected value of a parabolic spline function on a single sampling interval is:

$$
\begin{aligned}
& M_{x}[n]=\frac{1}{T} \int_{0}^{t_{d}}\left(a_{2}[n] t^{2}+a_{1}[n] t+a_{0}[n]\right) d t= \\
& =\frac{t_{d}}{T}\left(a_{2}[n] \frac{t_{d}^{2}}{3}+a_{1}[n] \frac{t_{d}}{2}+a_{0}[n]\right) .
\end{aligned}
$$

Accordingly, the expression for the second initial moment of the parabolic spline function on one sampling interval has the form:

$$
\begin{aligned}
& A_{2 x}[n]=\frac{1}{T} \int_{0}^{t_{d}}\left(a_{2}[n] t^{2}+a_{1}[n] t+a_{0}[n]\right)^{2} d t= \\
& =\frac{t_{d}}{T}\left(a_{2}[n] \frac{t_{d}^{4}}{5}+a_{2}[n] a_{1}[n] \frac{t_{d}^{3}}{2}+a_{1}^{2}[n] \frac{t_{d}^{2}}{3}+\right. \\
& \left.+a_{0}[n] a_{2}[n] \frac{2 t_{d}^{2}}{3}+a_{0}[n] a_{1}[n] t_{d}+a_{0}^{2}[n]\right),
\end{aligned}
$$

and the dispersion (the second central moment) has the form [11]:

$$
M_{2 x}[n]=A_{2 x}[n]-M_{x}^{2}[n] .
$$

If the parabolic spline - approximation of a harmonic signal on its period - is defined by $m$ discrete samples, then for a single sampling interval, the expected value is equal to:

$$
M_{x}=\frac{1}{m}\left[\sum_{n=1}^{m}\left(\frac{1}{3} a_{2}[n]+\frac{1}{2} a_{1}[n]+a_{0}[n]\right)\right],
$$

and the second initial moment is equal to:

$$
\begin{aligned}
& A_{2 x}=\frac{1}{m}\left(\sum_{n=1}^{m} \frac{1}{5} a_{2}^{2}[n]+\frac{1}{2} a_{1}[n] a_{2}[n]+\frac{1}{3} a_{1}^{2}[n]+\right. \\
& \left.+\frac{2}{3} a_{0}[n] a_{2}[n]+a_{0}[n] a_{1}[n]+a_{0}^{2}[n]\right) .
\end{aligned}
$$

For a cubic spline approximation of a harmonic signal for a unit sampling interval, the dispersion of the approximated signal is given by:

$$
\begin{aligned}
D_{x} & =\frac{1}{m} \sum_{n=1}^{m}\left\{\frac{1}{7} a_{3}^{2}[n]+\frac{1}{5}\left(2 a_{1}[n] a_{3}[n]+a_{2}^{2}[n]\right)+\right. \\
& +\frac{1}{2}\left(a_{0}[n] a_{3}[n]+a_{1}[n] a_{2}[n]\right)+ \\
& +\frac{1}{3}\left(2 a_{0}[n] a_{2}[n]+a_{2}[n] a_{3}[n]+a_{1}^{2}[n]\right)+ \\
& \left.+a_{0}[n] a_{1}[n]+a_{0}^{2}[n]\right\} .
\end{aligned}
$$

In particular, with 6 discrete intervals $(m=6)$ at the period of the signal, the dispersion value, found by means of (12), is $D_{x}=0.471$.

Comparing this value with the theoretical value (3), one can determine that the error of calculation of dispersion is equal $5.9 \%$.

Fig. 3 presents a plot of the dispersion relative determining error from the number $N$ of discrete intervals per period of the signal. It is seen from this figure that even when $m=10$, the accuracy of finding the dispersion is high enough (error 1\%).

The dispersion of the approximated signal is determined [11] similar to (11):

$$
M_{2 x}=A_{2 x}-M_{x}^{2} .
$$

In particular, with 8 samples per signal period $(\mathrm{m}=8)$, this dispersion is equal to:

$$
M_{2 x}=0.502 \text {. }
$$

This value corresponds quite accurately to a theoretical value determined from (3). 


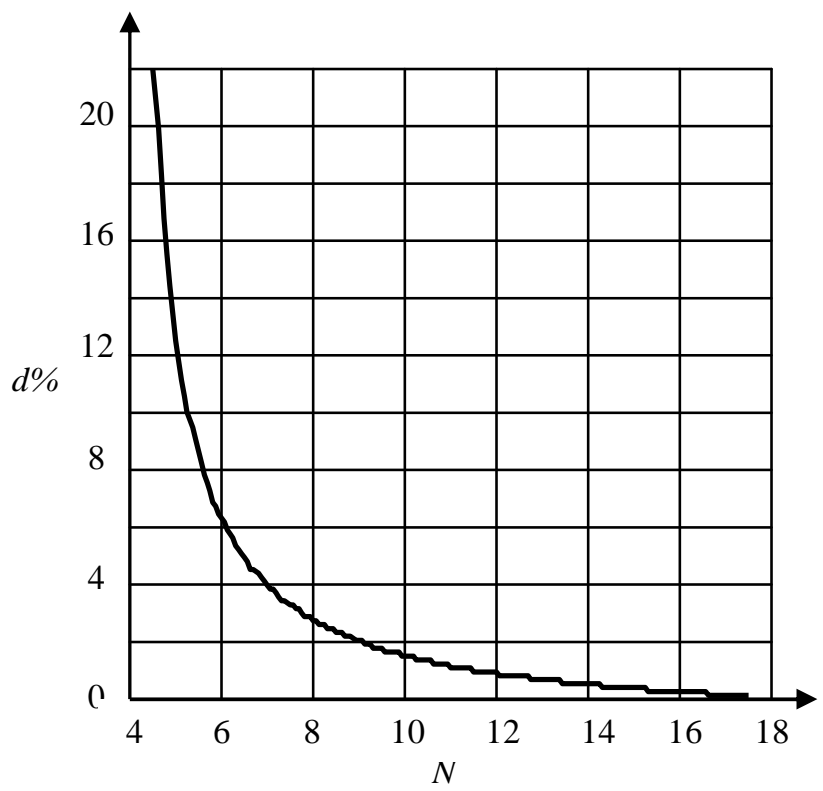

Fig. 3. Dependence of the relative error $d(\%)$ in determining the dispersion of harmonic signal using cubic spline approximation from the number $N$ of discrete intervals per period of the signal.

The third and the fourth initial moments are determined in a similar way:

$$
\begin{aligned}
& A_{3}=\frac{1}{m}\left[\frac{1}{4} a_{1}^{3}[n]+\frac{3}{2} a_{0}[n] a_{1}[n] a_{2}[n]+\right. \\
& +\frac{1}{7} a_{2}^{3}[n]+a_{0}^{3}[n]+\frac{3}{5} a_{1}^{2}[n] a_{2}[n]+a_{0}^{2}[n] a_{2}[n]+ \\
& +\frac{1}{2} a_{1}[n] a_{2}^{2}[n]+\frac{3}{2} a_{0}^{2}[n] a_{1}[n]+\frac{3}{5} a_{0}[n] a_{2}^{2}[n]+ \\
& \left.+a_{0}[n] a_{1}^{2}[n]+a_{1}^{2}[n] a_{0}[n]\right] ; \\
& A_{4}=\frac{1}{m}\left[\sum_{n=1}^{m} \frac{1}{5} a_{1}^{4}[n]+3 a_{0}^{2}[n] a_{1}[n] a_{2}[n]+\frac{1}{9} a_{2}^{4}[n]+\right. \\
& +\frac{12}{5} a_{0}[n] a_{1}^{2}[n] a_{2}[n]+2 a_{0}[n] a_{1}[n] a_{2}^{2}[n]+a_{0}^{4}[n]+ \\
& +\frac{1}{2} a_{1}[n] a_{2}^{3}[n]+\frac{6}{5} a_{0}^{2}[n] a_{2}^{2}[n]+2 a_{0}^{3}[n] a_{1}[n]+ \\
& +\frac{6}{7} a_{1}^{2}[n] a_{2}^{2}[n]+\frac{2}{3} a_{1}^{3}[n] a_{2}[n]+2 a_{0}^{2}[n] a_{1}^{2}[n]+ \\
& \left.+a_{0}[n] a_{1}^{3}[n]+\frac{4}{3} a_{0}^{3}[n] a_{2}[n]+\frac{4}{7} a_{2}^{3}[n] a_{0}[n]\right] .
\end{aligned}
$$

The corresponding equations (12) - (14) can be performed by various microprocessor based devices that are processing a vibro-acoustic signal. Such devices are part of modern systems that carry out sampling and analog-todigital signal conversion.

\section{SAMPLE OF CALCULATION}

The third and the fourth central moments of a vibroacoustic signal are determined by the expressions [11]

$$
\begin{aligned}
& M_{3}=A_{3}-3 M_{x} A_{2}+2 M_{x}^{3}, \\
& M_{4}=A_{4}-2 M_{x} A_{3}+6 M_{x}^{2} A_{2}-M_{x}^{4} .
\end{aligned}
$$

For eight sample sites for the period of a signal, the values of these moments are equal to:

$$
M_{3}=0.07, M_{4}=0.347 \text {. }
$$

Comparing these values with the theoretical, which are determined by (3) - (4), one can see that the error in these moments calculating does not exceed $7.5 \%$.

It is interesting to determine the dependence of the value of the fourth moment on the number $N$ of sampling points per signal period. This dependence is shown in Fig. 4.

It is clear that even with six sampling intervals over the period of the signal (i.e., a relatively rare sampling), the error in determining of the fourth central moment is in the order of $20 \%$, which is a good indicator for statistical measurements.

The use of other digital filters can lead to a decrease of an error in determining the statistical moments of the third and the fourth order of the vibroacoustic signal.

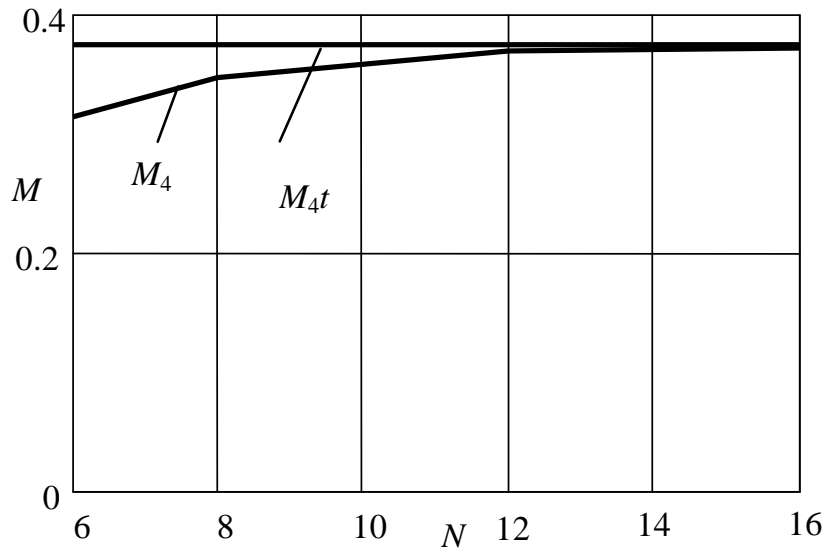

Fig. 4. Dependence of the fourth moment value on the number $N$ of sampling points per signal period: $M_{4}$ - the moment calculated for the approximated signal; $M_{4} t$ is the theoretical value of this moment.

For example, for a seven-point parabolic spline filter, the expressions for the spline-approximation coefficients are determined by the relations:

$$
\begin{aligned}
& a_{0}[n]=\frac{1}{64}(x[n-3]-6 x[n-2]+15 x[n-1]+44 x[n]+ \\
& +15 x[n+1]-6 x[n+2]+x[n+3]), \\
& a_{1}[n]=\frac{1}{32}(-x[n-3]+8 x[n-2]-29 x[n-1]+ \\
& \quad+29 x[n+1]-8 x[n+2]+x[n+3]), \\
& a_{2}[n]=\frac{1}{64}(x[n-3]-9 x[n-2]+37 x[n-1]-29 x[n]- \\
& -29 x[n+1]+37 x[n+2]-9 x[n+3]+x[n+4]) .
\end{aligned}
$$

In Fig. 5 there is the dependence of the determination errors of the fourth central moment on the sampling intervals number $N$ for parabolic spline approximation, using a seven-point (curve 2) and five-point digital filter (curve 1). 
$\delta, \%$

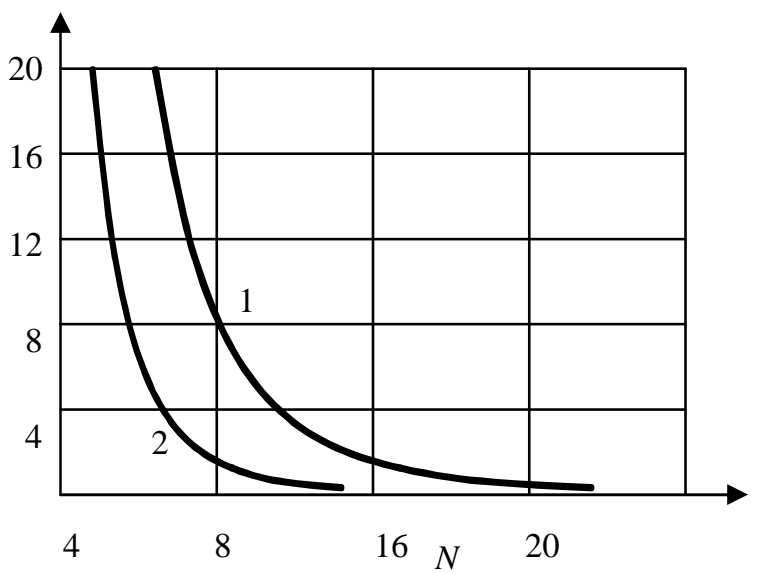

Fig. 5. Dependence of the error in determining the 4th central moment of the vibro-acoustic signal on sampling intervals number $N$ per period of the harmonic signal. 1- using a 5-point spline approximation, 2 - using a 7 point spline approximation.

From this figure, it is clearly seen that the use of a seven-point filter makes it possible to significantly increase the accuracy of the statistical characteristics, determined with a small number of sampling intervals per the period of the harmonic signal. Thus, for example, even at $N=8$, the error in determining the fourth central moment does not exceed $0.6 \%$.

The moment values determined by (12) - (14) are used in the equations for skewness (Skew) and Exess which can be used for early diagnosis of defects in friction pairs:

$$
\text { Skew }=\frac{M_{3}}{D_{x}^{3 / 2}} ; \quad E x=\frac{M_{4}}{D_{x}^{2}}-3 .
$$

Let us consider the influence of random noise imposed on a vibro-acoustic signal (2) on the accuracy of determining high-order statistical moments.

In this case, the vibration signal is determined by expression:

$$
x=\cos \omega t+k \cdot y(t),
$$

where $y(t)$ is a random signal with a uniform distribution density with intensity $k$.

Fig. 6 shows the dependence of the error in determining the Excess on the maximum value of the noise level, which is represented as a percentage of the signal amplitude. It can be seen from this figure that even at a 35\% level of a random component, the error in determining the excess is less than $10 \%$.

\section{RESULTS AND DISCUSSION}

An early diagnosis of nascent defects is possible by determining the statistical moments of the third and fourth orders of the vibro-acoustic signal. This diagnostics is possible in the real time operation of the controlled mechanism. To reduce the frequency of a vibro-acoustic signal sampling in determining its statistical moments, it is advisable to use a spline approximation of the discrete signal values.

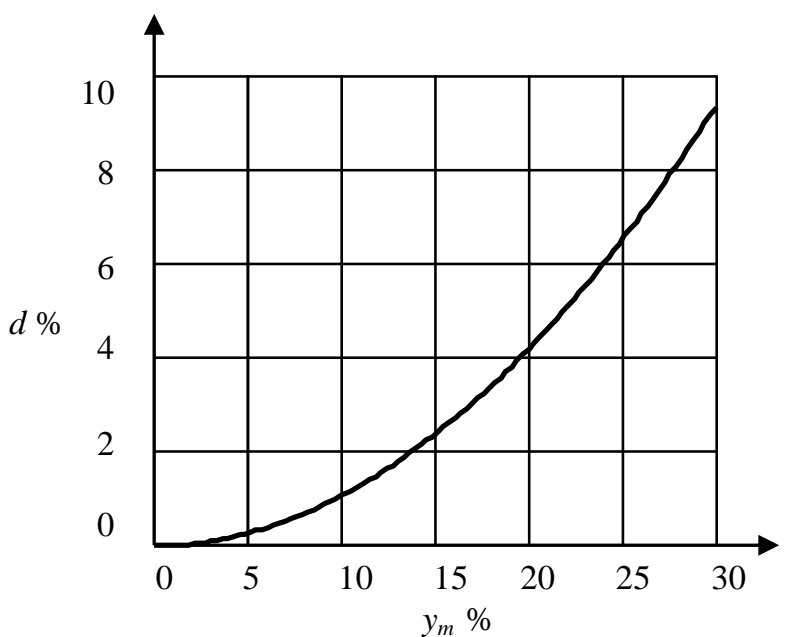

Fig. 6. Dependence of the error $d(\%)$ in determining the Excess of the signal at the level $y_{m}(\%)$ of the noise component

\section{CONCLUSION}

The coefficients of the spline-approximating function (parabolic or cubic) are determined quite simply using digital filtering algorithms, and these coefficients are used to calculate the statistical moments of 2,3 , and 4 orders of the vibro-acoustic signal. The use of cubic spline approximation makes it possible to improve the accuracy of determining the mathematical expectation and dispersion of a signal, but the use of parabolic spline approximation makes it possible to use simpler relations in determining the 4th order moment (Excess) with a sufficiently small error in its determination. The application of the proposed methods provides an insignificant influence of high-frequency random noise on the accuracy of determining of high-order statistical moments of a vibro-acoustic signal, which is due to the filtering properties of spline-approximation algorithms.

\section{References}

[1] V. Koshkin, Methods of measurement and experimental study of noise and vibration of car engines, Moscow: Mechanical engineering, 1988.

[2] V. Tolsky, Vibroacoustics of cars, Moscow: Mechanical engineering, 1988 .

[3] M. Genkin, Vibro-acoustic diagnostics of incipient defects, Moscow: Mechanical Engineering, 1984.

[4] C. Liefooghe,. Integration of structural dynamics into fatique prediction, Noise \& Vibration Worldwide, No.1, pp.6-8, 1992.

[5] T. Toutountzakis, C K Tan, D Mba, Application of Acoustic Emission to seeded gear fault detection, NDT \& E International, Volume 38, Issue 1, January 2005, pp 27-36.

[6] A. M. Al-Ghamdi, P. Cole, R Such, D. Mba, Estimation of bearing defect size with Acoustic Emission, INSIGHT, Vol. 46, no. 12, pp 758761, Dec 2004.

[7] D. Mba, A. Cooke, D. Roby, G. Hewitt, Detection of shaft-seal rubbing in large scale power generation turbines with Acoustic Emissions; Case study, Journal of power and energy, part A, I Mech E, Vol 218, No. 2 , Part A, pp. 71-82, March 2004.

[8] C. K. Tan, An investigation on the diagnostic and prognostic capabilities of acoustic emission (AE) on a spur gearbox, $\mathrm{PhD}$ Thesis, School of Engineering, Cranfield University, UK, 2005.

[9] A. M. Al-Ghamdi, D. Mba, A comparative experimental study on the use of Acoustic Emission and vibration analysis for bearing defect 
identification and estimation of defect size, Mechanical Systems and Signal Processing, Volume 20, Issue 7, pp. 1537-1571, October 2006.

[10] N. Baydar, A. Ball, Detection of incipient tooth defect in helical gears using multivariate statistics, Mechanical Systems and Signal Processing, 15 (2), pp. 303-321, 2001.

[11] Ash, Robert B. Basic propability theory, Robert B. Ash, Dover publications, New York, 2008.

[12] M.D. Genkin, A.G. Sokolova, Vibroacoustic diagnostics of machines and mechanisms, Moscow, Mashinostroenie publ., 1987, $288 \mathrm{p}$.

[13] T.J. Holroyd, N. Randall, Use of Acoustic Emission for Machine Condition Monitoring, British Journal of Non-Destructive Testing, 35(2), pp. 75-78, 1993.

[14] L. M. Roger, The application of vibration analysis and acoustic emission source location to on-line condition monitoring of antifriction bearings, Tribology International, 1979, pp. 51-59.

[15] Y. He, X. Zhang, M. Friswell, Defect Diagnosis for Rolling Element Bearings Using Acoustic Emission. Vibration and Acoustics, 131 (061012), pp. 1-10, 2009

[16] M. Elforjani, D. Mba, Accelerated natural fault diagnosis in slow speed bearings with acoustic Emission. Engineering Fracture Mechanics, 77, pp. 112-127, 2010.

[17] S. Al-Dossary, R.I. Hamzah, D. Mba, Observations of changes in acoustic emission waveform for varying seeded defect sizes in a rolling element bearing. Applied Acoustics, 70, pp. 58-81. 2009.

[18] Morhain, A., and Mba, D., Bearing defect diagnosis and acoustic Emission. Engineering Tribology, 217 (4), 257-272, 2003.

[19] D. Mba, and R.H. Bannister, Condition monitoring of low-speed rotating machinery using stress waves: Part 1 and Part 2, Journal of Process Mechanical Engineering, 213(3), pp. 153-185, 1999.

[20]D.G. Gu, J.G. Kim, Y.S. An, B.K..Choi, Detection of faults in gearboxes using acoustic emission signal. Journal of Mechanical Science and Technology, 25 (5), pp. 1279-1286, 2011.

[21] D. Mba, A. Cooke, D. Roby, G. Hewitt, „Detection of shaft-seal rubbing in largescale power generation turbines with Acoustic Emissions; Case study", Journal of power and energy - part A, I Mech E, Vol 218, No. 2, Part A, pp 71-82, ISSN 0957-6509, March 2004.

[22] C. K. Tan and D. Mba, „Identification of the Acoustic Emission source during a comparative study on diagnosis of a spur gearbox", Tribology International, Vol. 38, Issue 5, pp. 469-480, 2005.

[23] K. Bruzelius, D. Mba, „An initial investigation on the potential applicability of Acoustic Emission to rail track fault detection,.. NDT \& E International, 2004, Volume 37, Issue 7, pp. 507-516.

[24] L. Alfayez, D. Mba, „Detection of incipient cavitation and the best efficiency point of a $2.2 \mathrm{MW}$ centrifugal pump using Acoustic Emission, Journal of Acoustic Emission, Vol. 22, pp. 77-82, December 2004.

[25] T. Holroyd. „Acoustic Emission \& Ultrasonic“e, First Edition. Coxmoor Publishing Company, Oxford, 2000

26] R.I. Raja Hamzah, D. Mba, ,The influence of operating condition on acoustic emission (AE) generation during meshing of helical and spur gear", Tribology International, vol. 42. Iss. 1, pp. 3-14, 2009.

[27] P. Lange, Spline - approximation of discrete values of signals using digital filtering methods, Journal of Samara State Technical University, Ser. Physical and Mathematical Sciences, Issue 19, pp. $134-138,2003$.

[28] P. Lange, M. Ungarov, O. Matveev, Approximation of discrete values of signals using cubic spline filter, Journal of Samara State Technical University, Ser. Technical Sciences, Issue 2(50), pp. 106114, 2016.

[29] R. Hamming, Digital Filters. Prentice Hall inc., Englewood Cliffs, New Jersey, 1977, 224 p. 